\title{
Erratum to: Involvement of Cellular Prion Protein in $\alpha$-Synuclein Transport in Neurons
}

\author{
Laura Urrea ${ }^{1,2,3,4}$ - Miriam Segura-Feliu ${ }^{1,2,3,4}$ - Masami Masuda-Suzukake ${ }^{5}$ \\ Arnau Hervera ${ }^{1,2,3,4}$ • Lucas Pedraz ${ }^{6}$. José Manuel García-Aznar ${ }^{7}$. \\ Miquel Vila ${ }^{8,9}$ - Josep Samitier ${ }^{10,11,12}$ - Eduard Torrents ${ }^{6}$. \\ Isidro Ferrer $^{4,13,14,15}$ - Rosalina Gavín ${ }^{1,2,3,4}$ - Masato Hagesawa ${ }^{5}$. \\ José Antonio del Río ${ }^{1,2,3,4}$
}

Published online: 5 May 2017

(C) Springer Science+Business Media New York 2017

\section{Erratum to: Mol Neurobiol DOI 10.1007/s12035-017-0451-4}

The presentation Dr. José Manuel García-Aznar's name was incorrect. "García-Aznar" should be his Family Name. Citation should be "García-Aznar, JM" instead of "Aznar, JMG”. Also the two affiliations below of Dr. Josep Samitier were missing.

The online version of the original article can be found under doi:10.1007/ s12035-017-0451-4

José Antonio del Río

jadelrio@ibecbarcelona.eu

1 Molecular and Cellular Neurobiotechnology, Institute of Bioengineering of Catalonia (IBEC), Parc Científic de Barcelona, Baldiri Reixac 15-21, E-08028 Barcelona, Spain

2 Department of Cell Biology, Physiology and Immunology, Universitat de Barcelona, Barcelona, Spain

3 Center for Networked Biomedical Research on Neurodegenerative Diseases (CIBERNED), Barcelona, Spain

4 Institute of Neuroscience, University of Barcelona, Barcelona, Spain

5 Department of Dementia and Higher Brain Function, Tokyo Metropolitan Institute of Medical Science, Setagaya-ku, Tokyo 1568506, Japan

6 Bacterial infections: antimicrobial therapies, Institute of Bioengineering of Catalonia (IBEC), Parc Científic de Barcelona, Barcelona, Spain

7 Multiscale in Mechanical and Biological Engineering (M2BE), Aragon Institute of Engineering Research, Department of Mechanical Engineering, University of Zaragoza, Zaragoza, Spain
Department of Electronics, University of Barcelona, Martí i Franquès 1, E-08028 Barcelona, Spain.

Centro de Investigación Biomédica en Red en Bioingeniería, Biomateriales y Nanomedicina (CIBERBBN), 28029 Madrid, Spain.

With these, the authors hereby publish the corrected information as presented. The original article was also corrected.

8 Neurodegenerative Diseases Research Group, Vall d'Hebron Research Institute-Center for Networked Biomedical Research on Neurodegenerative Diseases, Autonomous University of Barcelona, Barcelona, Spain

9 Catalan Institution for Research and Advanced Studies (ICREA), Barcelona, Spain

10 Nanobioengineering Group, Institute for Bioengineering of Catalonia, (IBEC), Parc Científic de Barcelona, Barcelona, Spain

11 Department of Electronics, University of Barcelona, Martí i Franquès 1, E-08028 Barcelona, Spain

12 Centro de Investigación Biomédica en Red en Bioingeniería, Biomateriales y Nanomedicina (CIBER-BBN), 28029 Madrid, Spain

13 Institut de Neuropatologia, IDIBELL-Hospital Universitari de Bellvitge, Hospitalet de Llobregat, Spain

14 Departamento de Patologia y Terapeutica Experimental, Facultad de Medicina, Universidad de Barcelona, Barcelona, Spain

15 Centro de Investigación Biomédica en Red de Enfermedades Neurodegenerativas (CIBERNED), Barcelona, Spain 